\title{
Spectrum of Biopsy Proven Multisystemic Sarcoidosis in Pakistan: A Systemic Review
}

\author{
Murad R, Naeem A and Mubarak F* \\ Radiology Department, Aga Khan University, Pakistan
}

*Corresponding author: Fatima Mubarak, Radiology Department, Aga Khan University, Karachi, Pakistan, Tel: 03002550951; Email: fatima.mubarak@aku.edu

\section{Review Article}

Volume 4 Issue 2

Received Date: May 16, 2020

Published Date: June 17, 2020

DOI: $10.23880 /$ crij-16000171

\section{Abstract}

Sarcoidosis is a granulomatous disorder affecting multiple organs and systems however, its etiology is unknown. It is characterized by formation of non-caseating granulomas in the affected organs. The most common sites involved include lungs and the lymphoid system. Extrapulmonary involvement has also been reported with abdomen being the most common extrapulmonary site. Other systems involved include the skin, the eyes; the liver and spleen; the heart; the nervous system, musculoskeletal system, renal, and endocrine systems. This review article will help explore the different manifestations of sarcoidosis in various organs.

Keywords: Sarcoidosis; Toxins; Lung disease; Lymphadenopathy; Histopathology; Cranial Nerves

Abbreviations: CT: Computed Tomography; HRCT: High Resolution Computed Tomography; FDG-PET Fluorodeoxyglucose-Positron Emission Tomography; MRI: Magnetic Resonance Imaging; CSF: Cerebrospinal Fluid; MCP: Metacarpophalangeal Joints.

\section{Introduction and Background}

Sarcoidosis is an immune system disorder characterised by non-necrotising granulomas. Although the etiology of this condition is unclear, environmental and genetic factors may play some role in its pathogenesis [1]. It is a worldwide disease with incidence of $1-40$ cases per 100000 people per year and a prevalence of $0.2-64$ cases per 100000 people [1]. Diagnosis of sarcoidosis is generally based upon a relevant history, demonstration of non-caseating granulomas in more than one organ; measurement of angiotensin-converting enzyme level, the enzyme level is elevated in $40 \%$ to $90 \%$ of patients with active disease, negative staining and culture for acid fast bacilli, absence of exposure to toxins, and no history of drug-induced disease [2]. The pathological hallmark of the disease is the non-caseating sarcoid granuloma, composed of histiocytes surrounded by lymphocytes and mononuclear cells [3]. The clinical and radiological manifestations of the disease vary according to the system it affects. A wide variety of imaging features are found, which can resemble patterns of other pathological conditions. Therefore, the aim of this review article is to detail the most important clinical features and the main radiological findings of sarcoidosis in various organs, in order to simplify the identification of the disease process for the radiologists [4].

\section{Review}

\section{Pulmonary Involvement of Sarcoidosis}

Chest radiograph is considered as most common initial investigation to diagnose sarcoidosis [5]. The Scadding Classification Criteria for chest radiograph appearance remains widely used for the staging purpose.

Stage 0 is a normal radiograph.

Stage 1 is Mediastinal or Hilar lymphadenopathy.

Stage 2 is Lymphadenopathy and Lung parenchymal involvement.

Stage 3 is Parenchymal involvement only.

Stage 4 is pulmonary fibrosis. 


\section{Clinical Radiology \& Imaging Journal}

The presence of characteristic chest abnormalities on radiograph correlated with relevant history and laboratory investigations may be sufficient for diagnosis of sarcoidosis. However, if further evaluation is required, CT is usually performed, which is more sensitive for detection of parenchymal abnormalities and thoracic lymphadenopathy [1]. HRCT is more sensitive as compared to conventional CT for detection and assessment of subtle parenchymal lesions of lung structures [6]. HRCT also allows differentiation between reversible disease from irreversible lung disease and fibrosis, thus helping in prognosticating the disease leading towards accurate management. Several other imagings have also been used like MRI and FDG PET CT however; confirmation of non caseating granulomas on pathology is the lead towards the definitive diagnosis.

The most common findings on chest X-ray are lymphadenopathy associated/ not associated with nodules and infiltrates. The more advanced disease leads to pulmonary fibrosis [7]. The most typical pattern of pulmonary parenchymal involvement includes diffuse pulmonary perilymphatic micronodular infiltration with perilobular and fissural distribution with superior and posterior predominance [8]. The other characteristic findings encountered on CT are hilar and mediastinal lymphadenopathy, reticular patterns, mass densities, infiltrates, mosaic pattern, subpleural nodules and groundglass appearance. In last stage, traction bronchiectasis, honeycombing and cysts can be demonstrated [7]. The major complications of end stage pulmonary disease include pulmonary hypertenstion and chronic pulmonary aspergillosis [9] (Figures 1\&2).

The diagnosis of pulmonary sarcoidosis typically relies on findings on pulmonary function testing correlated with chest imaging and histopathology.

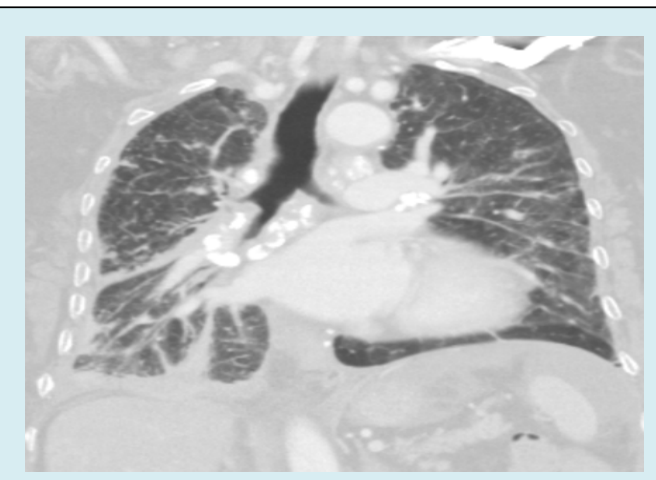

Figure 1: Multiple pleural based and peri-bronchovascular nodular densities with interlobar fissural thickening and nodularity.

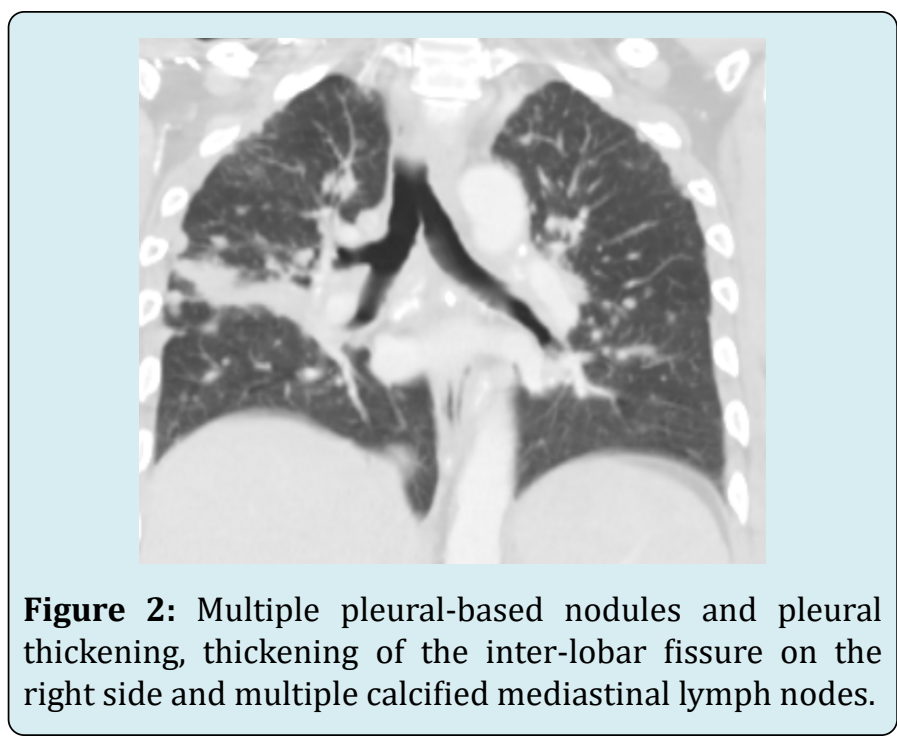

\section{Neurosarcoidosis}

Neurosarcoidosis occurs in $3 \%$ to $10 \%$ of patients with already known sarcoidosis. Cranial neuropathy and leptomeningeal involvement are the most common manifestations, but any part of the nervous system can be affected [10]. It can involve pachymeninges, brain parenchyma and ventricular system [11]. Hypothalamus and pituitary involvement can also be seen [12]. Spinal cord can also be rarely affected. Cranial nerves commonly involved are II, VII, and VIII [10] with cranial nerve II most frequently involved on imaging [11]. Thickening of involved cranial nerves is seen associated with post contrast enhancement suggesting epineural and perineural inflammatory changes. Clinical symptoms can be demonstrated according to which cranial nerve is involved.

Meningeal involvement is appreciated in $10-20 \%$ cases of neurosarcoidosis [10]. Typical symptoms of the affected patient include chronic headache and signs of meningeal irritation. Typical findings on CSF are: pleocytosis $\quad<220$ cells $/ \mathrm{mm}^{3}$ ) with lymphocytic predominance and/or raised protein $(<4.3 \mathrm{~g} / \mathrm{l})$. Reduced CSF glucose can also be seen [12]. Findings in brain parenchyma may include solitary/ multiple supra/infratentorial lesions which are likely secondary to spread of inflammation from prior involved leptomeninges along the Virchow-Robin spaces. The lesions characteristically appear iso-intense on T1-weighted images and show T2 prolongation, although intralesional haemorrhage may alter the appearance of lesion. Necrosis and calcification are rarely seen. Contrast enhancement pattern is variable.

Vascular and perivascular involvement inflammation can be demonstrated which can cause cerebral ischemia or 


\section{Clinical Radiology \& Imaging Journal}

parenchymal or subarachnoid hemorrhage [11]. However, recurrent ischemic stroke, haemorrhagic stroke and dural venous sinus thrombosis are infrequently reported. Sarcoidal granulomas can be found in intramedullary, intradural, and extradural portions of the spinal cord predominantly involving upper thoracic and cervical spinal cord. The most dominant findings are demonstrated in intramedullary location and lesions often extend over multiple segments showing T1/T2 prolongation and patchy post-contrast enhancement, which is often peripheral. Fusiform dilatation of affected cord segment is usually seen with overlying leptomeningeal enhancement [11]. Hypothalamic and pituitary involvement can be demonstrated as suprasellar inflammatory lesions. However, symptoms may arise from hypothalamic dysfunction, hypopituitarism or compression of optic chiasm by mass effect [12] (Figures $3 \& 4$ ).

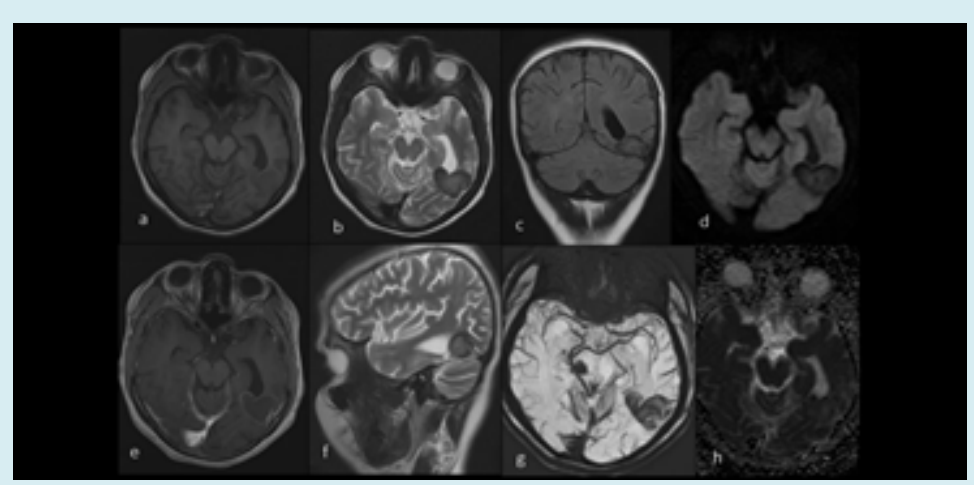

Figure 3: MRI brain images showing large lobulated focus within left temporal region returning T2 hypointense (b) and T1 isointense signals (a), it is showing signal dropout along its rim on SWI sequence (g), it is also showing minimal post contrast (e), Associated focal dural enhancement is also seen (e).

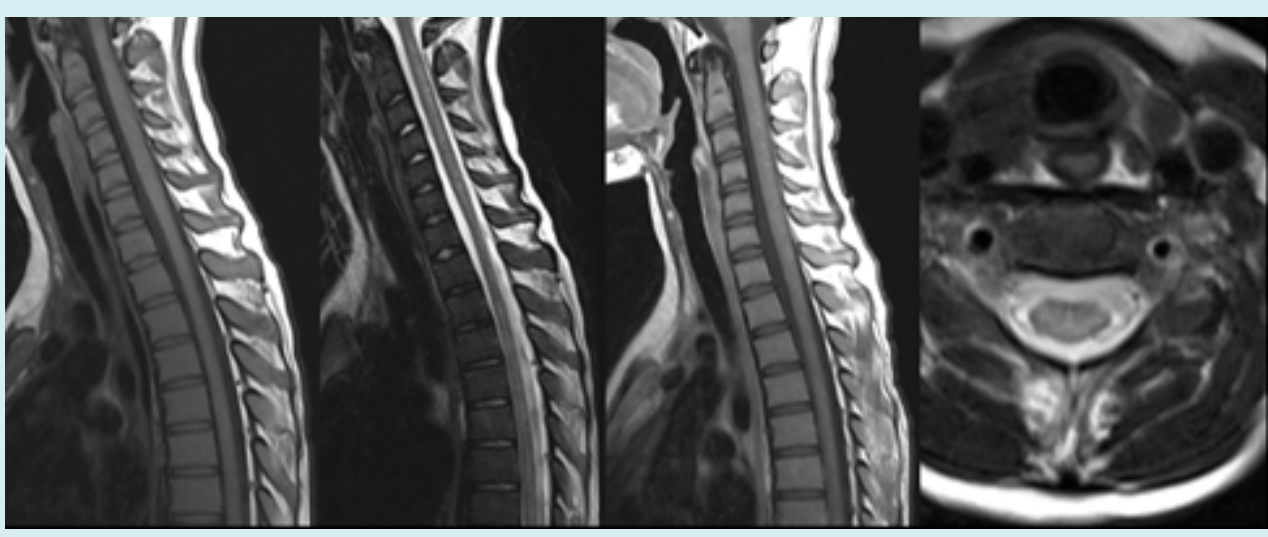

Figure 4: MRI cervical spine images in which cervical spinal cord is showing abnormal T2 hyperintense signal areas involving the anterior as well as posterior horns bilaterally throughout the extent of visualized spinal cord. These areas are showing subtle post contrast enhancement.

\section{Skeletal Sarcoidosis}

Bony sarcoidosis can be demonstrated myopathies, arthropathies and osseus pathologies.

Joints: Most identifiable manifestation is acute arthritis as a component of Lofgren's syndrome: Triad of joint pain, hilar lymphadenopathy and erythema nodosum, with bilateral ankle joints most commonly affected [13]. Subsequently, knees, wrist, elbows and MCP joints can also be involved. Imaging findings are usually demonstrated as periarticular soft tissue swelling and tenosynovitis [13]. Synovial biopsies show granulomas [14]. Chronic arthritis usually occurs with other extrapulmonary manifestations. There is bilateral symmetrical involvement of medium/large joints with joint space narrowing and demineralization of the subchondral bone.

Muscles: Skeletal muscle involvement is demonstrated in three patterns that are acute, chronic and nodular. Acute form is manifestated similarly to dermatomyositis; chronic form usually manifests as proximal muscle weakness; Nodular pattern manifests as palpable nodes, most commonly involving lower extremity. 


\section{Clinical Radiology \& Imaging Journal}

Bones: Three structural abnormalities can be appreciated in osseus pattern:

1. Permeative 'moth-eaten' appearance, with predominant involvement of the cortex of phalanges, usually accompanied by soft tissue swelling.

2. Lytic lesions (bone cysts), appearing as cortical defects in the phalangeal heads or round punch-out lesions.

3. Sclerotic lesions, which are demonstrated in the spine and are similar to those seen in metastatic disease.

Phalanges of both hands and feet, particularly the second and third middle and distal phalanges are markedly involved in skeletal sarcoidosis. Axial disease is represented as lytic or sclerotic lesions involving the vertebral bodies and can be appreciated in any segment however it is usually unilateral [14]. MRI often demonstrates no periosteal involvement or cortical destruction in large long bones; however, cortical disruption and extraosseous expansion are more frequently seen in small bones. The three basic MRI features which may help in distinguishing sarcoidosis from metastases are intralesional or perilesional fat, lesion border characteristics, and extraosseous mass; however, the sarcoid lesions cannot reliably be differentiated from metastasis on imaging. If the lesions are inconclusive, biopsy is the recommendation (Figure 5).

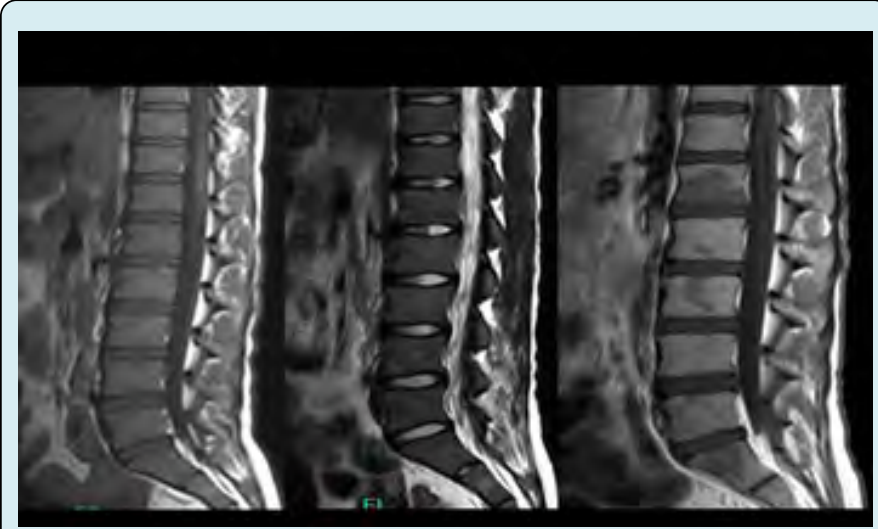

Figure 5: MRI lumbosacral spine images showing areas of non-enhancement in multilevel vertebral bodies, seen on post contrast image (c), these abnormal areas are isointense on non-contrast T1 and T2 images.

\section{Abdominal Sarcoidosis}

Abdomen is the most common extrapulmonary site to be affected by sarcoidosis with predominant involvement of liver and spleen [1]. Hepatomegaly is most common imaging finding associated and reported with hepatic sarcoidosis followed by nodular lesions which show characteristic hypointensity on all sequences of MRI, most apparent on T2-weighted fat-saturated images. Complications mostly involve increasing size of these granulomatous lesions leading to liver cirrhosis and portal hypertension. On MRI, contour irregularities of the liver and raised periportal signal intensity are usually appreciated. Spleen is affected in the very similar way as the hepatic involvement as evident by splenomegaly and multiple, hypoechoic, hypodense, hypointense, and nonenhancing lesions, scattered in the spleen parenchyma [2].

Other organs to be involved are:

Biliary Tree: Intra and extra hepatic with granulomas resulting in strictures and cholestasis.

Pancreas: Involvement may lead to pancreatitis as a complication.

Kidneys: Interstitial nephritis with an infiltrative pattern is typically appreciated. Abnormal calcium metabolism is also observed nephrocalcinosis, nephrolithiasis, and interstitial calcium deposition. These may lead to renal failure.

GIT: Stomach antrum is the most commonly affected ranging from simple mucosal thickening to granulomatous lesions. Small bowel is least affected and sigmoid colon is the most frequent site to be involved in large intestine presenting as multiple manifestations.

Peritoneum: Exudative ascites, single peritoneal nodular lesion or multiple granulomatous lesion can be visualized on imaging.

Lymph Nodes: Lymph nodes at porta hepatis, para-aortic region, and the celiac axis are most frequently affected. These are typically less than $20 \mathrm{~mm}$ in diameter and more discrete rather than confluent and show mild post contrast enhancement on MRI (Figure 6).

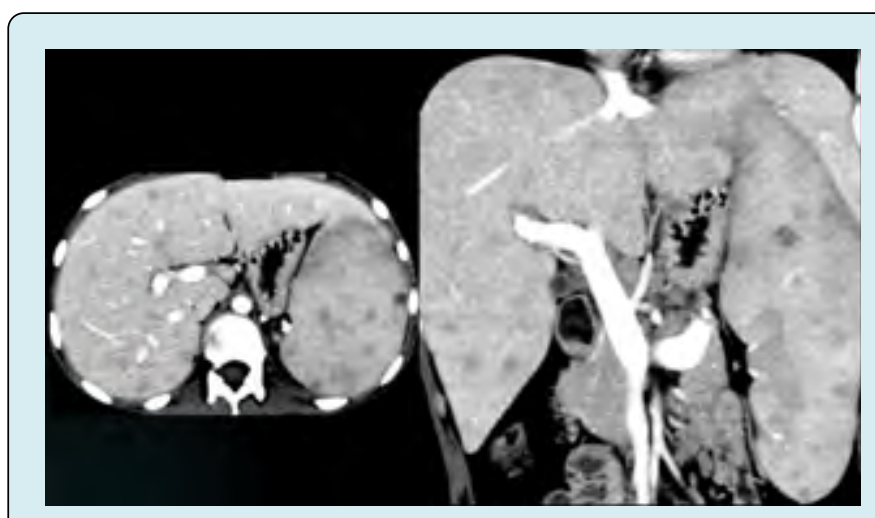

Figure 6: Axial and coronal CT abdomen post-contrast images showing hepatosplenomegaly with multiple hypodense areas of varying sizes are in both lobes of liver and spleen.

\section{Other Organs to be Involved by Sarcoidosis}

\section{Head and Neck Sarcoidosis}

The most frequent sites to be involved are orbital structures including globe, optic nerve sheath complex, 


\section{Clinical Radiology \& Imaging Journal}

lacrimal gland and extraocular muscles [15]. Amongst these ocular manifestations, the most common reported manifestation is anterior uveitis followed by intermediate and posterior uveitis [16]. Characteristic features on imaging are evident as thickening, nodularity and enhancement of involved structures. Other organs of head and neck region demonstrating manifestations of sarcoidosis include sinonasal cavities, larynx and salivary glands. The parotid gland shows most dominant involvement amongst salivary glands and demonstrates as painless enlargement of the gland.

\section{Cardiac Sarcoidosis}

Any part of cardiovascular system can be affected including atria, ventricles, valves, papillary muscles, pericardium, conduction system, and coronary vessels [17]. Patients are diagnosed with cardiac sarcoidosis when they have histopathological changes in other organs or clinical signs, together with cardiomegaly. Pericardial effusions and ventricular aneurysms may also be appreciated. Transthoracic echocardiography is recommended as the initial screening test in patients with systemic sarcoidosis. Typical imaging findings on Cardiac MR are: acute phase of the disease usually characterized by non-coronary distribution wall motion abnormalities, increased myocardial wall thickness, or increased intramyocardial signal intensity on T2-weighted images due to granulomatous lesion and edema associated with inflammation. Chronic phase may be demonstrated by myocardial wall thinning or aneurysm and a low signal on T2-weighted images but high late gadolinium enhanced signal intensity suggesting scaring or fibrosis. Definitive diagnosis requires pathology confirmation of noncaseating granuloma by endomyocardial biopsy, although the sensitivity is relatively poor.

\section{Cutaneous Sarcoidosis}

Lesions can range from minimal skin involvement to extensive disease that can result in significant morbidity. Although any site on the skin can be involved, including the mucosa, it preferentially occurs in sites of prior injury, such as scars and tattoos [18]. Skin manifestations can be categorized into specific lesions and nonspecific lesions: specific manifestations include presence of noncaseating granulomas observed within skin biopsies, and nonspecific lesions are considered reactive to systemic sarcoidosis however, they do not have granulomas on biopsy [18]. Certain skin manifestations can provide clues to underlying organ involvement and potential prognostic information, which can help towards therapeutic decisions. Systemic evaluation for underlying organ involvement by sarcoidosis is of supreme importance.

\section{Conclusion}

Sarcoidosis can be presented in different manner with variety of clinical manifestations and imaging patterns. The diagnosis can be established with adequate evaluation of clinical manifestations correlated with imaging patterns and laboratory findings with definite confirmation by detection of non-caseating granulomas on biopsy and histopathology after the exclusion of other granulomatous disorders.

\section{References}

1. Ganeshan D, Menias CO, Lubner MG, Pickhardt PJ, Sandrasegaran K, et al. (2018) Sarcoidosis from head to toe: what the radiologist needs to know. Radiographics 38(4): 1180-200.

2. Gezer NS, Başara I, Altay C, Harman M, Rocher L, et al. (2015) Abdominal sarcoidosis: cross-sectional imaging findings. Diagnostic and Interventional Radiology 21(2): 111-117.

3. Balan A, Hoey ET, Sheerin F, Lakkaraju A, Chowdhury FU, et al. (2010) Multi-technique imaging of sarcoidosis. Clinical Radiology 65(9): 750-760.

4. Palmucci S, Torrisi SE, Caltabiano DC, Puglisi S, Lentini $\mathrm{V}$, et al. (2016) Clinical and radiological features of extra-pulmonary sarcoidosis: a pictorial essay. Insights imaging 7(4): 571-587.

5. Escalon JG, Lynch DA (2018) Imaging features of sarcoidosis. QJM: An International J Medic 111(9): 649651.

6. Dhagat PK, Singh S, Jain M, Singh SN, Sharma RK (2017) Thoracic Sarcoidosis: imaging with high resolution computed tomography. J Clin Diagn Res 11(2): 15-18.

7. Baughman RP, Lower EE, Gibson K (2012) Pulmonary manifestations of sarcoidosis. La Presse Médicale 41(6): 289-302.

8. Jeny F, Bernaudin JF, Aubart FC, Brillet PY, Bouvry D, et al. (2019) Diagnosis issues in sarcoidosis. Respiratory Medicine and Research 77: 37-45.

9. Spagnolo P, Rossi G, Trisolini R, Sverzellati N, Baughman RP, Wells AU et al. (2018) Pulmonary sarcoidosis. The Lancet Respiratory Medicine 6(5): 389-402.

10. Ungprasert P, Matteson EL (2017) Neurosarcoidosis. Rheumatic Disease Clinics 43(4): 593-606.

11. Bathla G, Watal P, Gupta S, Nagpal P, Mohan S, Moritani $\mathrm{T}$, et al. (2018) Cerebrovascular manifestations of neurosarcoidosis: an underrecognized aspect of the 


\section{Clinical Radiology \& Imaging Journal}

imaging spectrum. AJNR 39(7): 1194-1200.

12. Ibitoye RT, Wilkins A (2017) Scolding NJ Neurosarcoidosis: a clinical approach to diagnosis and management. Journal of Neurology 264(5): 1023-1028.

13. Bechman K, Christidis D, Walsh S, Birring SS, Galloway J, et al. (2018) A review of the musculoskeletal manifestations of sarcoidosis. Rheumatology 57(5): 777-783.

14. Crawford B, Badlissi F, Calderón SA (2018) Orthopaedic considerations in the management of skeletal sarcoidosis. JAAOS 26(6): 197-203.
15. Chapman MN, Fujita A, Sung EK, Siegel C, Nadgir RN, et al. (2017) Sarcoidosis in the head and neck: an illustrative review of clinical presentations and imaging findings. American Journal of Roentgenology 208(1): 66-75.

16. Badhey AK, Kadakia S, Carrau RL, Iacob C, Khorsandi A, et al. (2015) Sarcoidosis of the head and neck. Head Neck Pathol 9(2): 260-268.

17. Tan JL, Fong HK, Birati EY, Han Y (2019) Cardiac sarcoidosis. The American journal of cardiology 123(3): 513-522.

18. Wanat KA, Rosenbach M (2015) Cutaneous sarcoidosis. Clinics in chest medicine 36(4): 685-702. 